\title{
Symptomatic Characteristics of Parkinson's Disease Induced by Neuroleptic Drugs, Based on a Functional Neuroimaging Diagnosis
}

\author{
Kei Tachibana, Keita Matsuura, Akihiro Shindo, Hirofumi Matsuyama, Yuichiro Ii, \\ Akira Taniguchi and Hidekazu Tomimoto
}

\begin{abstract}
:
Objective When patients take neuroleptics, the distinction between Parkinson's disease (PD) and druginduced parkinsonism (DIP) based solely on clinical features can become difficult. At present, ${ }^{123}$ I-FP-CIT SPECT (DAT-SPECT) and ${ }^{123}$ I-metaiodobenzylguanidine (MIBG) myocardial scintigraphy are widely used to supplement the differential diagnosis of parkinsonism. This study assessed the clinical symptoms and neurological findings in the patients suspected of having DIP based on DAT-SPECT findings.

Methods Twenty-three patients (11 men, 12 women, age: 52-81 years old) presenting with DIP were recruited. All patients underwent neurological examinations, including brain magnetic resonance imaging and DAT-SPECT. Patients who showed abnormal DAT-SPECT results underwent MIBG myocardial scintigraphy.

Results Eleven patients showed a reduction in the ligand uptake on DAT-SPECT (DAT-positive group), and nine of these patients showed a low delayed heart-to-mediastinum (H/M) ratio on MIBG myocardial scintigraphy. The remaining 12 patients showed normal results on DAT-SPECT (DAT-negative group). All patients in the DAT-positive group had asymmetric motor symptoms, whereas only 4 in the DAT-negative group exhibited this clinical feature $(\mathrm{p}=0.001)$. A detailed medical history showed that 7 of the 11 patients in the DAT-positive group had prodromal symptoms for PD. However, only 1 patient in the DAT-negative group exhibited these symptoms $(\mathrm{p}=0.009)$. Although two patients in the DAT-negative group showed poor improvement, they showed a normal H/M ratio on MIBG and no response to levodopa.

Conclusion The patients in the DAT-positive group might have prodromal symptoms that were worsened by neuroleptic drugs. The results of detailed history-taking and neurological findings seem to indicate cases of compromised dopaminergic transmission before the administration of neuroleptic drugs.
\end{abstract}

Key words: Parkinson disease, drug induced parkinsonism, asymmetric, autonomic dysfunction, tremor, dopamine transporter single-photon emission computed tomography

(Intern Med 59: 485-490, 2020)

(DOI: 10.2169/internalmedicine.2553-18)

\section{Introduction}

Parkinson's disease (PD) is a common neurodegenerative disorder characterized by rigidity, bradykinesia, tremor, and disturbances in gait as well as non-motor symptoms that are associated with dopaminergic system dysfunction. Neuroleptic treatments, including dopamine receptor antagonists, can often cause symptoms that resemble those seen in PD, thus leading to what is called "drug-induced parkinsonism" (DIP).

DIP is one of the most common causes of Parkinson'slike symptoms, second only to PD itself $(1,2)$. Unlike PD, however, previous studies have suggested that DIP is less likely to lead to resting tremor and more likely to manifest in symmetric symptoms as well as dyskinesia or akathisia. Furthermore, the symptoms associated with DIP appear to be less responsive to levodopa than those caused by 
PD $(3,4)$. While diagnosing DIP based on the patient's drug history alone may appear straightforward, it may be possible for DIP to mask the onset of PD, which can be provoked by neuroleptics (5).

Recently, ${ }^{123} \mathrm{I}$-FP-CIT SPECT (DAT-SPECT) and ${ }^{123} \mathrm{I}-$ metaiodobenzylguanidine (MIBG) myocardial scintigraphy have become widely used in medical practice to help distinguish between PD and DIP (5-8). Assessing neurologically related symptoms may be useful for differentiating patients with PD from those with pure DIP. Indeed, PD may be preceded by symptoms, such as subtle motor and non-motor features (9), including rapid eye movement sleep behavior disorder (RBD), olfactory dysfunction, and constipation (10).

In the present study, we sought to assess whether or not patients with DIP and PD could be differentiated from those with pure DIP based on DAT-SPECT abnormalities (DATpositive versus DAT-negative group). We compared the clinical features between DAT-positive and DAT-negative groups and also sought to confirm whether or not a decreased uptake of the MIBG myocardial scintigraphy ligand would occur invariably in the DAT-positive group.

\section{Materials and Methods}

Twenty-three patients (11 men, 12 women; age: 52-81 years old) who had developed parkinsonism after at least 2 weeks of neuroleptic treatment were enrolled in the study between August 2013 and July 2019. No patient had a prior history of parkinsonism, but all patients that were included in the study had a prior psychiatric diagnosis of schizophrenia, depression, or undetermined psychiatric disease. This study was approved by the Ethics Committee of Mie University Hospital (permit numbers 3148 and 2184), and all procedures were in accordance with the Declaration of Helsinki.

The patients were asked to report any history of medication, the time between the beginning of the neuroleptic treatment and the start of their symptoms (duration to worse/onset), any history of diabetes mellitus or heart disease, and any non-motor preclinical symptoms, including constipation, hyposmia, and RBD. Constipation was defined as stool defecation less than every other day or, if the patient consumed laxative drugs, less than on a daily basis. Hyposmia was assessed by questioning the patients on their ability to perceive odors and by directly assessing olfaction with freshly brewed coffee. Olfactory dysfunction was assessed with an odor stick identification test designed for Japanese people (OSIT-J) for two patients (11). Patients were screened for RBD by checking for the presence of a violent or excessive episode of movement during sleep. Motor symptoms, including tremor, rigidity, and bradykinesia, as well as the laterality of the symptoms were assessed independently by two neurologists. When both of them reported laterality of either tremor or rigidity, the presence of laterality was determined. During this assessment period, patients were not taking any anti-parkinsonian drugs.

Non-motor symptoms, including autonomic dysfunction and cognitive impairment, were also assessed. For the assessment of cognitive impairment, patients with a MiniMental State Examination (MMSE) score of $\leq 23$ were considered cognitively impaired. Urinary dysfunction was assessed using a questionnaire on urinary urgency and nocturia. Orthostatic hypotension was defined by a $30-\mathrm{mmHg}$ decrease in systolic blood pressure on standing. All patients underwent neuroimaging of the head with magnetic resonance imaging (MRI) and angiography (MRA) as well as DAT-SPECT; the latter was reviewed according to the visual assessment method of Kahraman et al., based on five predefined patterns (12). Each patient's DAT-SPECT findings were also independently assessed by two neurologists. The asymmetry index (AI) of the specific binding ratio (SBR) was calculated using the following equation, based on previous publications $(13,14)$ : AI of $\mathrm{SBR}=(\mathrm{SBR}$ of the right SBR of the left $) \times 2 /($ SBR of the right + SBR of the left $) \times$ $100[\%]$.

DAT-SPECT results were assessed as previously described (15). Patients who exhibited abnormal DAT-SPECT patterns (Grade 1-4) underwent an MIBG myocardial scintigraphy examination (16). Furthermore, eight patients who showed normal DAT-SPECT results also underwent MIBG myocardial scintigraphy. Using a standardized assessment method of MIBG myocardial scintigraphy (17), we defined a normal heart-to-mediastinum $(\mathrm{H} / \mathrm{M})$ ratio (delayed) as $\geq$ 2.20 and a low $\mathrm{H} / \mathrm{M}$ ratio as $<2.20$ (delayed) (18). In addition, we divided patients into two groups (PD or DIP) based on whether or not they exhibited a combined abnormal pattern on DAT-SPECT and whether or not they showed a low $\mathrm{H} / \mathrm{M}$ ratio on MIBG myocardial scintigraphy.

\section{Statistical analyses}

A univariate analysis and Mann-Whitney U-tests were used to compare continuous variables between the DATpositive and DAT-negative groups, while Fisher's exact test was used to analyze categorical data. Continuous variables are presented as the mean \pm standard deviation, whereas categorical variables are reported as percentages. A $p$ value $<0.05$ was considered statistically significant. Statistical analyses were performed using SPSS version 24 (IBM, Armonk, USA).

\section{Results}

Patients were divided into two groups based on whether their DAT-SPECT findings were normal $(\mathrm{n}=12)$ or abnormal $(\mathrm{n}=11)$ (DAT-positive group, Table 1$)$. Nine of the 11 patients in the DAT-positive group showed a low delayed H/ $\mathrm{M}$ ratio on MIBG myocardial scintigraphy (Figure A, B), while the remaining 2 showed a normal H/M ratio. A diagnosis of PD was made based on the DAT-SPECT findings. Of the 12 DAT-negative patients, 7 showed a normal H/M ratio on MIBG myocardial scintigraphy (Figure C, D, Ta- 


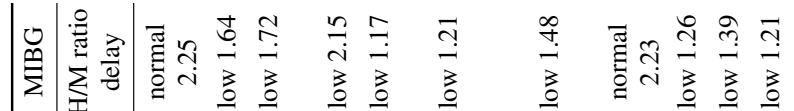

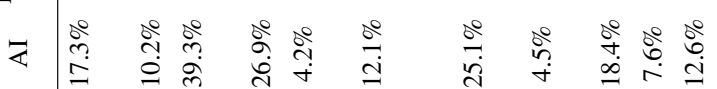

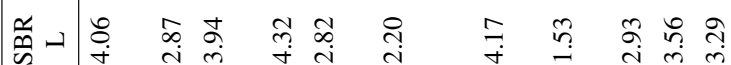

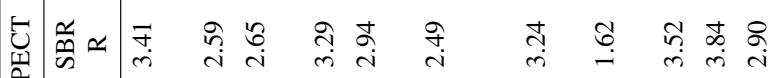

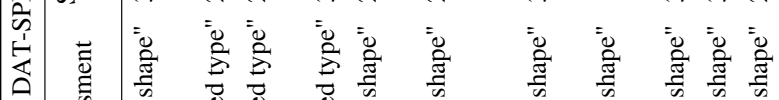

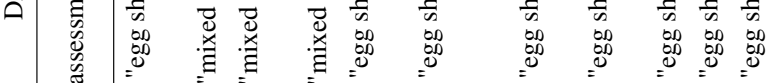
急告

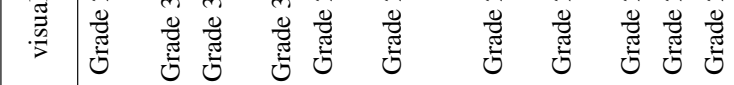

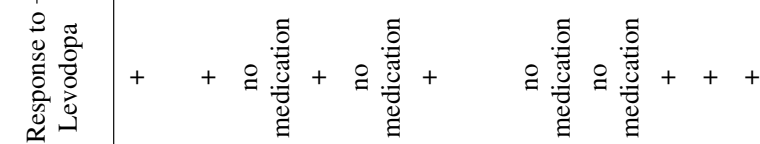

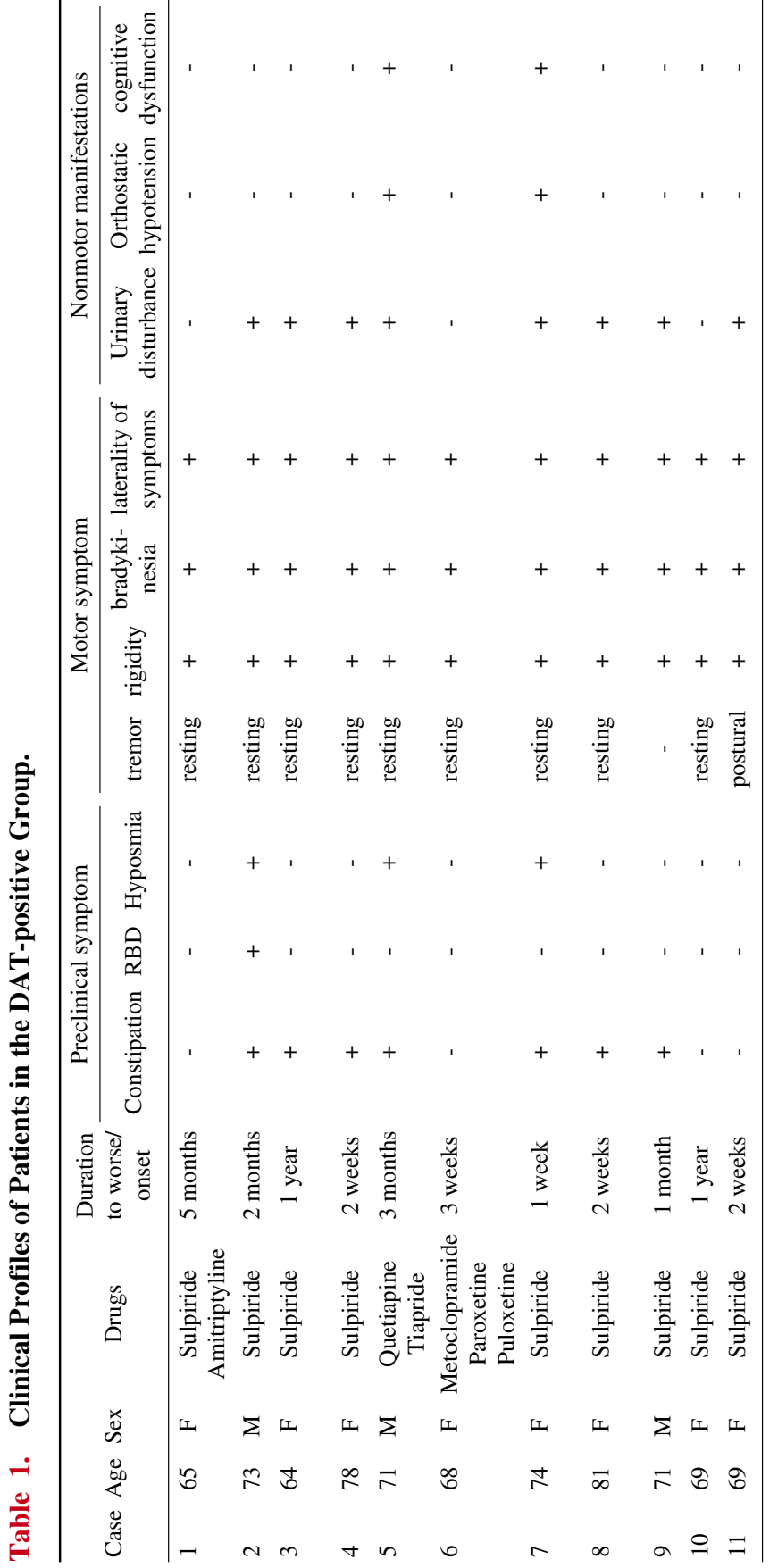

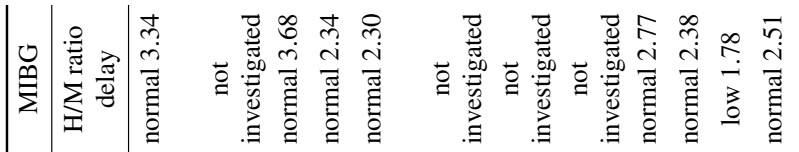
飞

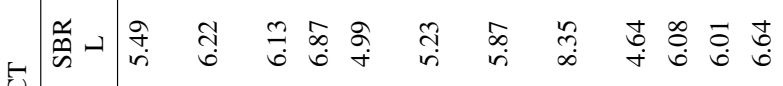

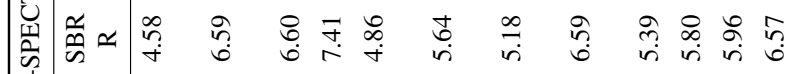

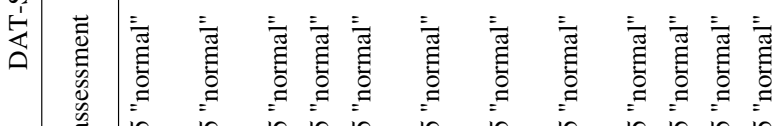
is in in in in in in in in in

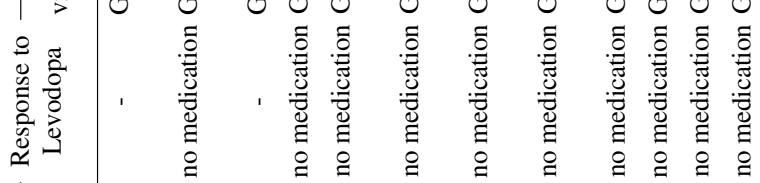

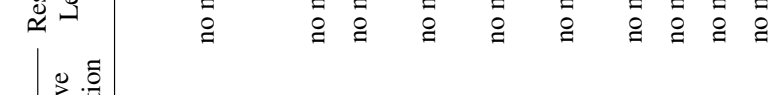

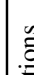

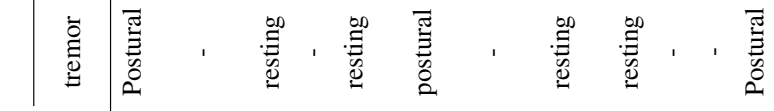

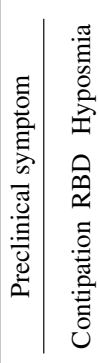

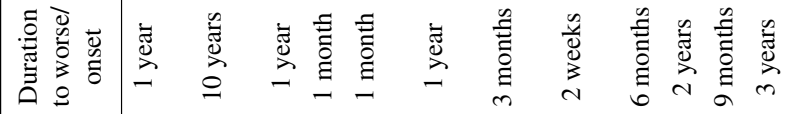

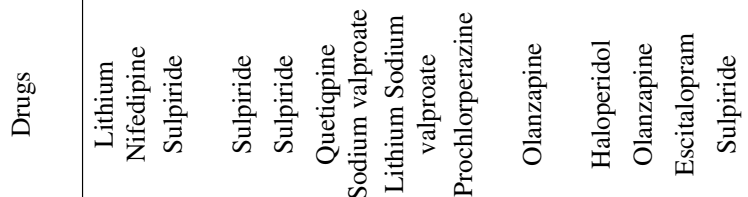

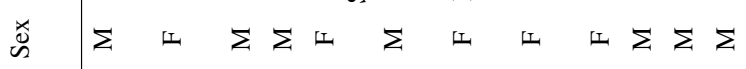

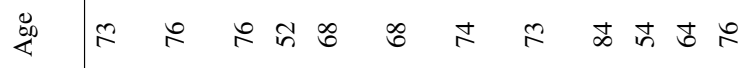

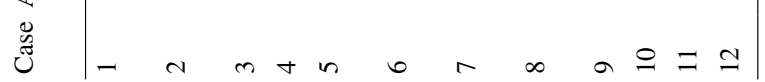




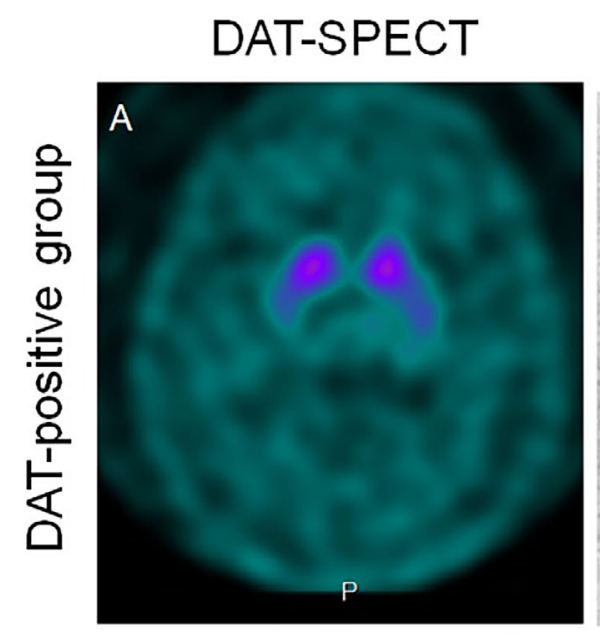

\section{MIBG myocardial scintigraphy}
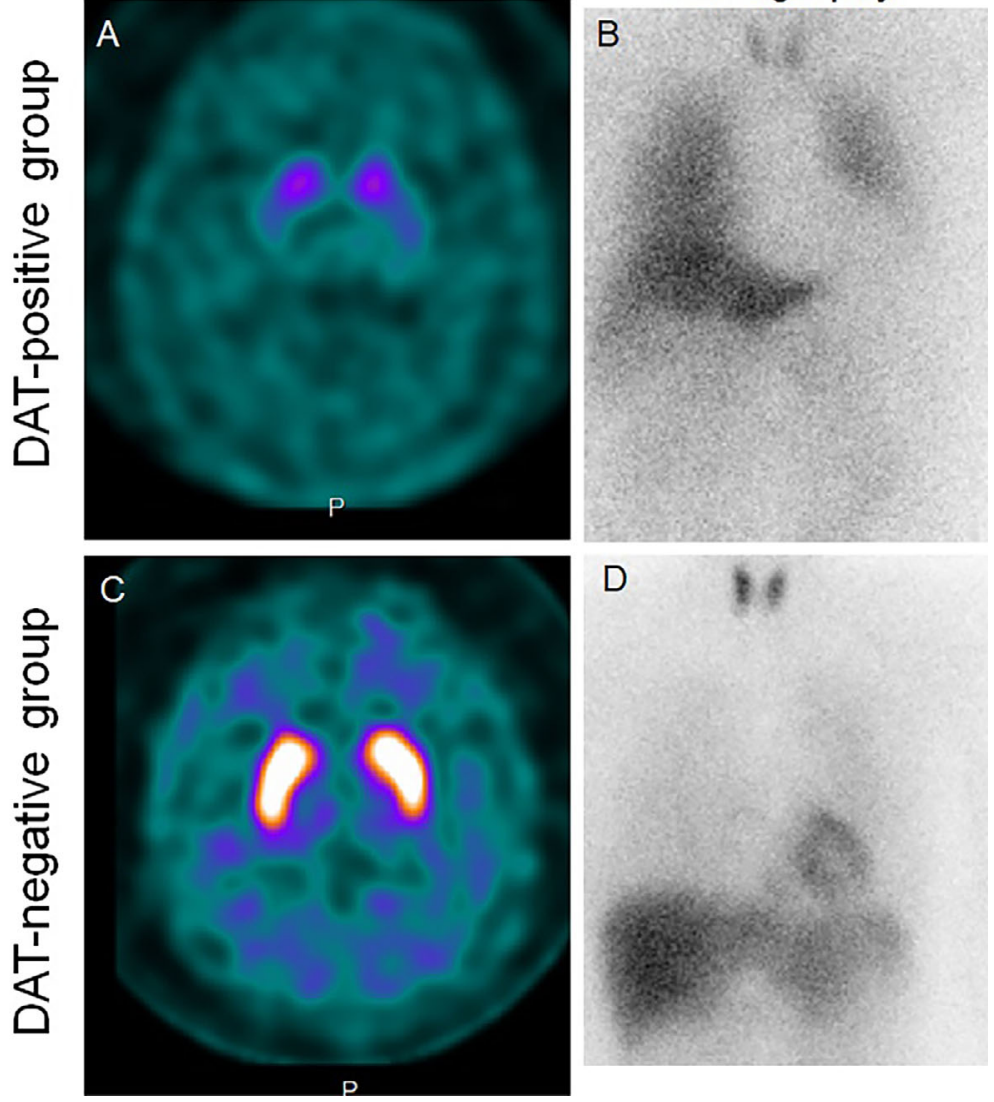

Figure. Representative images of the DAT-positive and DAT-negative groups. The DAT-positive group showed abnormal results on DAT-SPECT (A) and a decreased delay in the MIBG uptake on myocardial scintigraphy (B). These findings indicate PD. The DAT-negative group, in contrast, showed normal results on DAT-SPECT (C) and a normal delayed MIBG uptake (D), indicating DIP.

Table 3. Summary of Clinical Data in Patients with PD and DIP.

\begin{tabular}{lccc}
\hline & $\mathrm{PD}$ & $\mathrm{DIP}$ & \multirow{2}{*}{$\mathrm{p}$ value } \\
\cline { 2 - 3 } & $\mathrm{N}=11$ & $\mathrm{~N}=12$ & \\
\hline Patients & & & \\
Age, years & $71.2 \pm 5.1$ & $69.8 \pm 9.4$ & 1.000 \\
Sex, Male(M), Female(F) & $\mathrm{M} 3, \mathrm{~F} 8$ & $\mathrm{M} 7, \mathrm{~F} 4$ & 0.140 \\
Duration, month & $3.4 \pm 4.5$ & $19.7 \pm 33.3$ & $0.027^{*}$ \\
Preclinical symptom & & & \\
$\quad$ Constipation & $7(63.6 \%)$ & $1(9.1 \%)$ & $0.009^{*}$ \\
$\quad$ RBD & $1(9.1 \%)$ & $0(0 \%)$ & 0.478 \\
$\quad$ Hyposmia & $3(27.3 \%)$ & $0(0 \%)$ & 0.093 \\
Motor symptom & & & \\
$\quad$ Tremor & $10(90.9 \%)$ & $6(54.5 \%)$ & 0.145 \\
$\quad$ Resting & $9(81.8 \%)$ & $3(27.3 \%)$ & $0.036^{*}$ \\
$\quad$ Postural & $1(9.1 \%)$ & $3(27.3 \%)$ & 0.590 \\
$\quad$ Rigidity & $11(100 \%)$ & $11(100 \%)$ & 1.000 \\
$\quad$ Bradykinesia & $11(100 \%)$ & $7(63.6 \%)$ & 0.093 \\
Laterality of symptom & $11(100 \%)$ & $4(36.4 \%)$ & $0.001^{*}$ \\
Non-motor symptom & & & \\
Urinary disturbance & $8(72.7 \%)$ & $3(27.3 \%)$ & $0.039^{*}$ \\
Orthostatic hypotension & $2(18.2 \%)$ & $0(0 \%)$ & 0.217 \\
Cognitive impairment & $2(18.2 \%)$ & $0(0 \%)$ & 0.217 \\
\hline
\end{tabular}

ble 2). One patient (case 11) who had a slightly low H/M ratio was also diagnosed with DIP, as his symptoms showed recovery after he stopped taking escitalopram, and his DATSPECT results were normal. No patient took any tricyclic antidepressants. A total of three patients had diabetes mellitus: case 2 in the DAT-positive group and cases 3 and 7 in the DAT-negative group. Furthermore, three patients had angina pectoris: case 5 in the DAT-positive group and cases 4 and 7 in the DAT-negative group. No patient from either group had significant vascular lesions on brain MRI or MRA.

We failed to detect a significant statistical difference between the DAT-positive and DAT-negative group with regard to age, sex, or non-motor symptoms, such as urinary disturbance, orthostatic hypotension, or cognitive impairment (Table 3). The duration to worsening/onset was longer in the DAT-negative group than in the DAT-positive group $(\mathrm{p}=$ 0.027). Seven patients in the DAT-positive group had at least one symptom among constipation, RBD, and hyposmia, whereas only 1 patient in the DAT-negative group exhibited any of these symptoms $(\mathrm{p}=0.009)$. We only tested cases 1 and 9 in the DAT-negative group for hyposmia, using the OSIT-J, and found their scores to be normal (7 and 8 , re- 
spectively) (11). Although there was no statistically significant difference between the DAT-positive and DAT-negative group in measures such as tremor $(\mathrm{p}=0.145)$ and postural tremor $(\mathrm{p}=0.590)$, the rate of resting tremor was significantly higher in the DAT-positive group than in the DAT-negative group ( $\mathrm{p}=0.036$ ). Furthermore, there were no significant differences in the occurrence of rigidity or bradykinesia. While all 11 patients in the DAT-positive group showed significant asymmetry, only 4 showed asymmetry in the DAT-negative group ( $\mathrm{p}=0.001)$. Urinary dysfunction was significantly more frequent in the DAT-positive group $(\mathrm{p}=0.039)$, while there were no significant differences in the occurrence of nonmotor symptoms, such as orthostatic hypotension and cognitive impairment.

During follow-up of the patients, seven in the DATpositive group still had symptoms and recovered after taking levodopa. In contrast, 10 patients in the DAT-negative group improved after stopping neuroleptics, while 2 did not improve. In addition, levodopa treatment failed to improve their symptoms.

\section{Discussion}

This study supports the notion that certain clinical features, such as constipation, resting tremor, asymmetry of motor symptoms, and urinary disturbance, may help differentiate PD from pure DIP. Furthermore, two patients in the DAT-negative group whose condition did not improve even after quitting neuroleptics may have had drug-induced secondary neuronal damage.

While DIP is characterized by the rapid onset of symmetric symptoms, the absence of tremor or akathisia, and the occurrence of bucco-linguo-masticatory dyskinesias $(3,19)$, clinically diagnosing patients with DIP can be difficult, as some symptoms overlap with PD (20). Furthermore, DIP may progress slowly or remit in approximately $10 \%$ of cases, even after the discontinuation of neuroleptic drugs. Interestingly, PD and DIP appear to have some distinct neuropathological features. For example, presynaptic dopaminesecreting neurons are diminished in PD (21) but are intact in patients with pure DIP (22). Therefore, antagonizing dopaminergic receptor transmission in patients with PD may accelerate and worsen their symptoms-a feature not shared by pure DIP, where the dopaminergic nerve terminals are intact. From a clinical perspective, patients with DIP and PD may exhibit resting tremors, asymmetry of motor symptoms, and urinary dysfunctions not found in pure DIP, as well as constipation.

Regarding these modes of discriminating patients with pure DIP from those with DIP and PD, it is important to consider tests that can help predict which patients may be more receptive to levodopa treatment and which may be more susceptible to the detrimental impact of neuroleptics in precipitating PD. Furthermore, the availability of functional neuroimaging versus the accuracy of clinical neurological examinations in differentiating PD from pure DIP may influ- ence future clinical practice.

Neurologists sometimes encounter patients with DIP whose symptoms do not seem to improve even after the neuroleptic drugs are discontinued. Neuroleptic drugs themselves not only block dopaminergic receptors but also inhibit the production of neurotrophic factors and neurotoxic free radicals (23). Interestingly, Foubert-Samier et al. showed that neuroleptics can increase the long-term risk of PD (24). In accordance with this possibility, our results showed the maintenance of $\mathrm{H} / \mathrm{M}$ ratios on MIBG myocardial scintigraphy in 2 of 11 patients in the DAT-positive group, although low $\mathrm{H} / \mathrm{M}$ ratios are less commonly observed in early-stage PD than late-stage PD (25). Furthermore, while olfactory dysfunction has been reported in approximately $90 \%$ of patients with early-stage PD (26), only a quarter of the patients in our DAT-positive group showed hyposmia. While we have no clear explanation for these results, they may indicate secondary dopaminergic neuronal damage induced by neuroleptic drugs.

This study has several limitations. First, our sample size was small, and future studies using a larger cohort may thus be warranted. Second, the patients in the DAT-positive group in our study showed parkinsonism after just a short duration on neuroleptic medication. However, previous studies have shown that patients only show these symptoms after neuroleptic treatment for several months $(5,27)$. While the effect observed here may have been driven by chance, it is also possible that the patients with PD in our clinical sample were particularly susceptible to neuroleptic drugs. Third, the number of patients who showed RBD in the DAT-positive group in our study was small; however, the frequency of RBD has previously been reported to be between $33 \%$ and $60 \%$ in patients with $\operatorname{PD}(28,29)$. This emphasizes the above-mentioned point that findings from small sample sizes need to be interpreted with caution. Fourth, most patients in the DAT-positive group showed a low $\mathrm{H} / \mathrm{M}$ ratio on $\mathrm{MIBG}$ myocardial scintigraphy. As mentioned earlier, however, almost half of patients in the early stages of PD show a normal H/M ratio. This discrepancy may be another result of the small number of patients included in this study. Furthermore, we were able to perform MIBG myocardial scintigraphy in only 8 of the 12 patients in the DAT-negative group. Among these eight patients, seven showed normal results on MIBG myocardial scintigraphy. Two patients showed no response to levodopa treatment, and their symptoms showed little recovery. There is a possibility that some of the remaining four patients would have shown a low $\mathrm{H} / \mathrm{M}$ ratio on MIBG myocardial scintigraphy. Fifth, we were only able to evaluate hyposmia using the OSIT-J, except for in two patients. The OSIT-J is a useful tool for examinations of Japanese PD patients (30). Further studies will therefore be needed for the evaluation of hyposmia.

\section{Conclusion}

This study shows that patients with PD can be differenti- 
ated from those with DIP based on an assessment that combines DAT-SPECT and motor symptom asymmetry. This approach may help clinicians decide whether or not to administer neuroleptics to patients with suspected PD. Furthermore, our data suggest that neuroleptic drugs may induce dopaminergic neuronal damage.

The authors state that they have no Conflict of Interest (COI).

\section{Acknowledgement}

The authors thank Yoshinori Hirata, MD, Akane Mizutani, MD, Natsuko Kato, MD, Hidehiro Ishikawa, MD, Ko Matsuo, MD, Takuya Shimada, MD, Masaru Asahi, MD, and Masayuki Sato, MD for assessing and treating the patients included in this study. We also thank the Mie University Radioisotope Center staff for their help.

\section{References}

1. Thanvi B, Treadwell S. Drug induced parkinsonism: a common cause of parkinsonism in older people. Postgrad Med J 85: 322326, 2009.

2. Lopez-Sendon J, Mena MA, de Yebenes JG. Drug-induced parkinsonism. Expert Opin Drug Saf 12: 487-496, 2013.

3. Hassin-Baer S, Sirota P, Korczyn AD, et al. Clinical characteristics of neuroleptic-induced parkinsonism. J Neural Transm (Vienna) 108: 1299-1308, 2001.

4. Sethi KD, Zamrini EY. Asymmetry in clinical features of druginduced parkinsonism. J Neuropsychiatry Clin Neurosci 2: 64-66, 1990.

5. Lorberboym M, Treves TA, Melamed E, Lampl Y, Hellmann M, Djaldetti R. [123I]-FP/CIT SPECT imaging for distinguishing drug-induced parkinsonism from Parkinson's disease. Mov Disord 21: 510-514, 2006.

6. Ba F, Martin WR. Dopamine transporter imaging as a diagnostic tool for parkinsonism and related disorders in clinical practice. Parkinsonism Relat Disord 21: 87-94, 2015.

7. Yoshii F, Ryo M, Baba Y, Koide T, Hashimoto J. Combined use of dopamine transporter imaging (DAT-SPECT) and (123) Imetaiodobenzylguanidine (MIBG) myocardial scintigraphy for diagnosing Parkinson's disease. J Neurol Sci 375: 80-85, 2017.

8. Brigo F, Erro R, Marangi A, Bhatia K, Tinazzi M. Differentiating drug-induced parkinsonism from Parkinson's disease: an update on non-motor symptoms and investigations. Parkinsonism Relat Disord 20: 808-814, 2014.

9. Berg D, Postuma RB, Adler CH, et al. MDS research criteria for prodromal Parkinson's disease. Mov Disord 30: 1600-1611, 2015.

10. Langston JW. The Parkinson's complex: parkinsonism is just the tip of the iceberg. Ann Neurol 59: 591-596, 2006.

11. Saito S, Ayabe-Kanamura S, Takashima Y, et al. Development of a smell identification test using a novel stick-type odor presentation kit. Chem Senses 31: 379-391, 2006.

12. Kahraman D, Eggers C, Schicha H, Timmermann L, Schmidt M. Visual assessment of dopaminergic degeneration pattern in 123IFP-CIT SPECT differentiates patients with atypical parkinsonian syndromes and idiopathic Parkinson's disease. J Neurol 259: 251260, 2012.

13. Dickson JC, Tossici-Bolt L, Sera T, et al. The impact of recon- struction method on the quantification of DaTSCAN images. Eur J Nucl Med Mol Imaging 37: 23, 2009.

14. Kuya K, Shinohara Y, Miyoshi F, Fujii S, Tanabe Y, Ogawa T. Correlation between neuromelanin-sensitive MR imaging and (123) I-FP-CIT SPECT in patients with parkinsonism. Neuroradiology 58: 351-356, 2016.

15. Matsuda $\mathrm{H}$, Murata M, Mukai $Y$, et al. Japanese multicenter database of healthy controls for [(123) I] FP-CIT SPECT. Eur J Nucl Med Mol Imaging 45: 1405-1416, 2018.

16. Orimo S, Ozawa E, Nakade S, Sugimoto T, Mizusawa H. (123) Imetaiodobenzylguanidine myocardial scintigraphy in Parkinson's disease. J Neurol Neurosurg Psychiatry 67: 189-194, 1999.

17. Nakajima K, Okuda K, Matsuo S, et al. Standardization of metaiodobenzylguanidine heart to mediastinum ratio using a calibration phantom: effects of correction on normal databases and a multicentre study. Eur J Nucl Med Mol Imaging 39: 113-119, 2012.

18. Nakajima K, Okuda K, Yoshimura $M$, et al. Multicenter crosscalibration of I-123 metaiodobenzylguanidine heart-tomediastinum ratios to overcome camera-collimator variations. J Nucl Cardiol 21: 970-978, 2014.

19. Hirose G. Drug induced parkinsonism. J Neurol 253: iii22-iii24, 2006.

20. Pieters LE, Bakker PR, van Harten PN. Asymmetric drug-induced parkinsonism and psychopathology: a prospective naturalistic study in long-stay psychiatric patients. Front Psychiatry 9: 18, 2018.

21. Gelb DJ, Oliver E, Gilman S. Diagnostic criteria for Parkinson disease. Arch Neurol 56: 33-39, 1999.

22. Shuaib UA, Rajput AH, Robinson CA, Rajput A. Neurolepticinduced Parkinsonism: clinicopathological study. Mov Disord 31: 360-365, 2016

23. Erro R, Bhatia KP, Tinazzi M. Parkinsonism following neuroleptic exposure: a double-hit hypothesis? Mov Disord 30: 780-785, 2015.

24. Foubert-Samier A, Helmer C, Perez F, et al. Past exposure to neuroleptic drugs and risk of Parkinson disease in an elderly cohort. Neurology 79: 1615-1621, 2012.

25. Nagayama H, Hamamoto M, Ueda M, Nagashima J, Katayama Y. Reliability of MIBG myocardial scintigraphy in the diagnosis of Parkinson's disease. J Neurol Neurosurg Psychiatry 76: 249-251, 2005.

26. Doty RL. Olfaction in Parkinson's disease. Parkinsonism Relat Disord 13 (Suppl 3): S225-S228, 2007.

27. Tinazzi M, Ottaviani S, Isaias IU, et al. [123I] FP-CIT SPET imaging in drug-induced Parkinsonism. Mov Disord 23: 1825-1829, 2008.

28. Boeve BF, Silber MH, Saper CB, et al. Pathophysiology of REM sleep behaviour disorder and relevance to neurodegenerative disease. Brain 130: 2770-2788, 2007.

29. Yoritaka A, Ohizumi H, Tanaka S, Hattori N. Parkinson's disease with and without REM sleep behaviour disorder: are there any clinical differences? Eur Neurol 61: 164-170, 2009.

30. Iijima M, Kobayakawa T, Saito $S$, et al. Smell identification in Japanese Parkinson's disease patients: using the odor stick identification test for Japanese subjects. Intern Med 47: 1887-1892, 2008.

The Internal Medicine is an Open Access journal distributed under the Creative Commons Attribution-NonCommercial-NoDerivatives 4.0 International License. To view the details of this license, please visit (https://creativecommons.org/licenses/ by-nc-nd/4.0/).

(C) 2020 The Japanese Society of Internal Medicine Intern Med 59: 485-490, 2020 